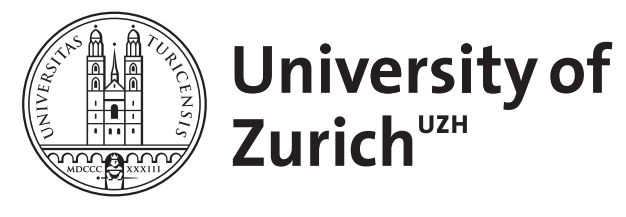

\title{
Aortic arch malformations
}

Kellenberger, C J

\begin{abstract}
Although anomalies of the aortic arch and its branches are relatively uncommon malformations, they are often associated with congenital heart disease. Isolated lesions may be clinically significant when the airways are compromised by a vascular ring. In this article, the development and imaging appearance of the aortic arch system and its various malformations are reviewed.
\end{abstract}

DOI: https://doi.org/10.1007/s00247-010-1607-9

Posted at the Zurich Open Repository and Archive, University of Zurich

ZORA URL: https://doi.org/10.5167/uzh-45492

Journal Article

Published Version

Originally published at:

Kellenberger, C J (2010). Aortic arch malformations. Pediatric Radiology, 40(6):876-884.

DOI: https://doi.org/10.1007/s00247-010-1607-9 


\title{
Aortic arch malformations
}

\author{
Christian J. Kellenberger
}

Received: 6 February 2010 /Accepted: 8 February 2010/Published online: 31 March 2010

(C) Springer-Verlag 2010

\begin{abstract}
Although anomalies of the aortic arch and its branches are relatively uncommon malformations, they are often associated with congenital heart disease. Isolated lesions may be clinically significant when the airways are compromised by a vascular ring. In this article, the development and imaging appearance of the aortic arch system and its various malformations are reviewed.
\end{abstract}

Keywords Aorta $\cdot$ Arteries Congenital malformation . MRI C CT Child

\section{Introduction}

A wide spectrum of congenital anomalies can occur during the formation of the aortic arch, brachiocephalic arteries and ductus arteriosus, most of them asymptomatic and merely an anatomic variant observed at imaging, but some with significant cardiovascular or respiratory symptoms requiring therapy. In this review, development of the aortic arch system, specific malformations and their imaging evaluation are discussed.

\section{Development of the aortic arch system and malformations}

Knowledge of the normal development of the aortic arch and its major branches helps us to understand and

\section{J. Kellenberger $(\square)$}

Department of Diagnostic Imaging,

University Children's Hospital,

Steinwiesstrasse 75 ,

CH 8032 Zürich, Switzerland

e-mail: christian.kellenberger@kispi.uzh.ch classify the various forms of aortic arch malformations $[1,2]$. During fetal development, six pairs of primitive aortic arches are sequentially formed connecting the truncus arteriosus and aortic sac with the paired dorsal aortae (Fig. 1). As successive arches develop, the previous arches regress with remnants eventually contributing to parts of the head and neck vessels. The aortic sac forms the ascending aorta, proximal aortic arch and the innominate artery. Remnants of the first arches form parts of the maxillary arteries; the second arches are parts of the stapedial arteries; and the third arches form the common carotid and a portion of the internal carotid arteries. The major persistent arches in humans are the fourth and sixth arches while the fifth arches usually regress completely. Derivates of the fourth arches contribute to a portion of the definitive normal left aortic arch between the left common carotid and subclavian arteries and the proximal portion of the right subclavian artery. The proximal portions of the sixth arches become the mediastinal segments of the pulmonary arteries while the distal portions form the ductus arteriosi. Although the right ductus arteriosus almost always disappears, a patent ductus arteriosus can persist on either side connecting the respective pulmonary artery to the aortic arch in the region of the subclavian artery origin or to the innominate artery at the origin of the subclavian artery on the side opposite to the aortic arch. The seventh cervical intersegmental arteries, caudal branches of the dorsal aortae, migrate cranially to a position opposite the fourth arches and become part of the subclavian arteries. The paired dorsal aortae fuse progressively from caudal to cranial. The left dorsal aorta normally forms the distal aortic arch and descending aorta while the right dorsal aorta regresses except for its proximal portion remaining part of the right subclavian artery. 


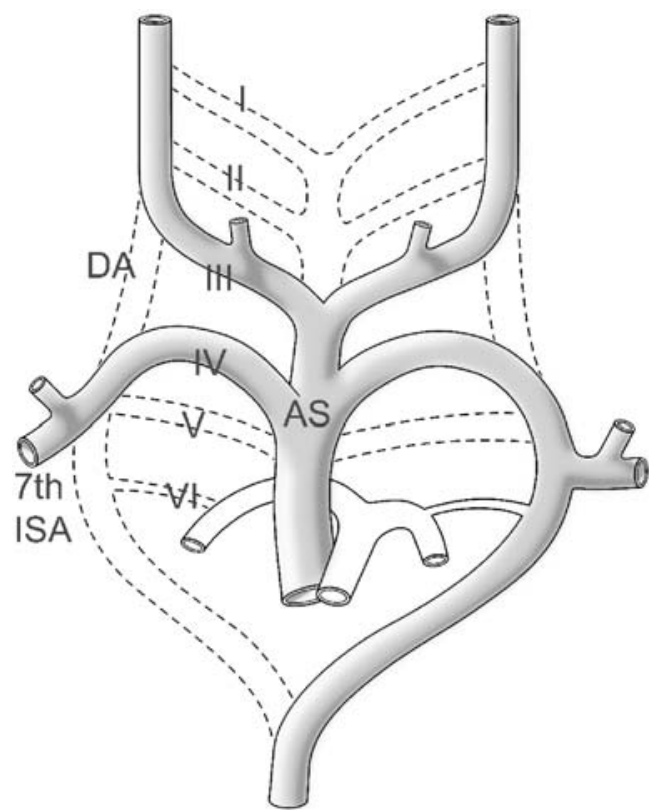

Fig. 1 Drawing illustrating the six pairs of aortic arches (I-VI) formed between the aortic sac $(A S)$ and paired dorsal aortae $(D A)$. Parts of the dorsal aortae and aortic arches that normally have disappeared at 7 weeks are shown with broken lines. ISA, intersegmental artery

Most malformations of the aortic arch can be explained by the hypothetical double arch described by Edwards [3, 4] and result from failure of regression, or regression in an abnormal site of the fourth aortic arch system. The potential sites of regression in the right and left arches are shown in Fig. 2 and will be referred to in the following discussion of the individual malformations.

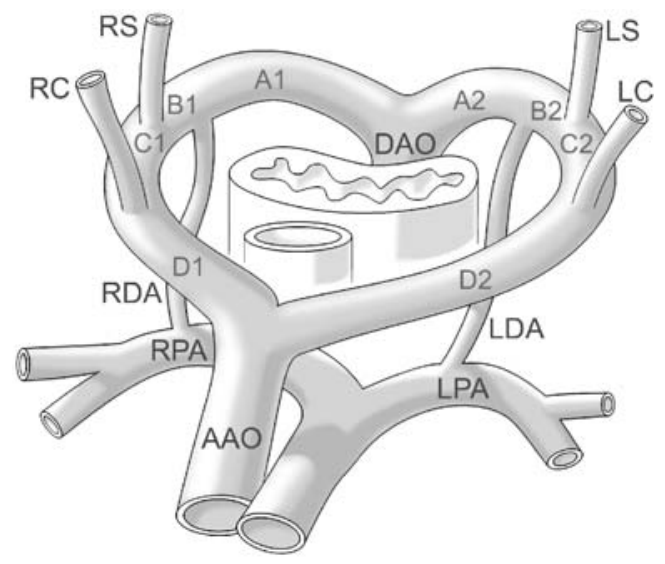

Fig. 2 Drawing illustrating Edwards' hypothetical double aortic arch encircling the trachea and oesophagus. $A 1-D 1$, potential sites of regression in the right aortic arch; $A 2-D 2$, potential sites of regression in the left aortic arch; $A A O$, ascending aorta; $D A O$, descending aorta; $R C$, right common carotid artery; $R S$, right subclavian artery; $L C$, left common carotid artery; $L S$, left subclavian artery; $R D A$, right ductus arteriosus; $L D A$, left ductus arteriosus; $R P A$, right pulmonary artery; $L P A$, left pulmonary artery
Left aortic arch

Normally, the right fourth aortic arch regresses distal to the origin of the right subclavian artery (A1) resulting in a left aortic arch that lies to the left of the trachea, arches over the left mainstem bronchus and continues as the descending aorta to the left of the spine. The first arterial branch passes to the right as the innominate artery, giving rise to the right common carotid and subclavian arteries, followed by the left common carotid and left subclavian arteries. The ductus arteriosus, either patent or ligamentous, is usually also on the left connecting the proximal left pulmonary artery with the distal aortic arch.

Numerous anatomic variations in the branching pattern of the brachiocephalic arteries exist with separate origins of common carotid arteries, subclavian arteries and vertebral arteries directly from the arch, or with joined origins of immediate neighbour arteries. A common trunk giving rise to the right innominate artery and left common carotid artery, and a separate origin of the left vertebral artery from the aortic arch proximal to the left subclavian artery, are quite frequent variants both with an incidence of about $10 \%$.

If the right fourth aortic arch regresses between the right common carotid artery and right subclavian artery (C1), a left aortic arch with aberrant origin of the right subclavian artery as last branch results. Such an aberrant right subclavian artery (Arteria lusoria) courses behind the oesophagus and constitutes an incomplete vascular ring seldom causing symptoms (Fig. 3). In the rare case where a right-sided ductus arteriosus between the right pulmonary artery and the right subclavian artery was present during the fetal period, the aberrant subclavian artery may originate from a retro-oesophageal aortic diverticulum (Kommerell diverticulum), which forms a complete vascular ring together with the right-sided ductus ligament.

If the right descending aorta persists together with a left aortic arch, the distal portion of the arch passes behind the oesophagus, which is called a circumflex aorta. Depending on the location of regression distal (B1) or proximal (C1) to the right subclavian artery, the brachiocephalic arteries branch normally or there is an aberrant right subclavian artery. If the ductus arteriosus is on the right side, the left circumflex aortic arch and the ductus ligament form a vascular ring.

Right aortic arch

A right aortic arch is rare in normal individuals and usually is associated with congenital cardiac malformations such as persistent truncus arteriosus, tetralogy of Fallot, and pulmonary atresia with ventricular septal defect. Regression in the left fourth aortic arch results in a right arch lying to the right side of the trachea, passing over the right mainstem bronchus and generally continuing as right descending aorta located to the right of the spine. 
Fig. 3 a, b Aberrant RS in a 9-year-old girl with chronic cough. Frontal chest radiograph (a) clearly shows that the aortic arch and descending aorta are on the left, with the aortic knob (asterisk) slightly indenting the trachea and the para-aortic stripe (arrowheads) located to the left of the spine. Frontal projection (b) of barium oesophagogram shows an oblique radiolucent band extending from the aortic arch (asterisk) inferiorly to the contralateral side superiorly. Findings are caused by the aberrant RS coursing behind the oesophagus
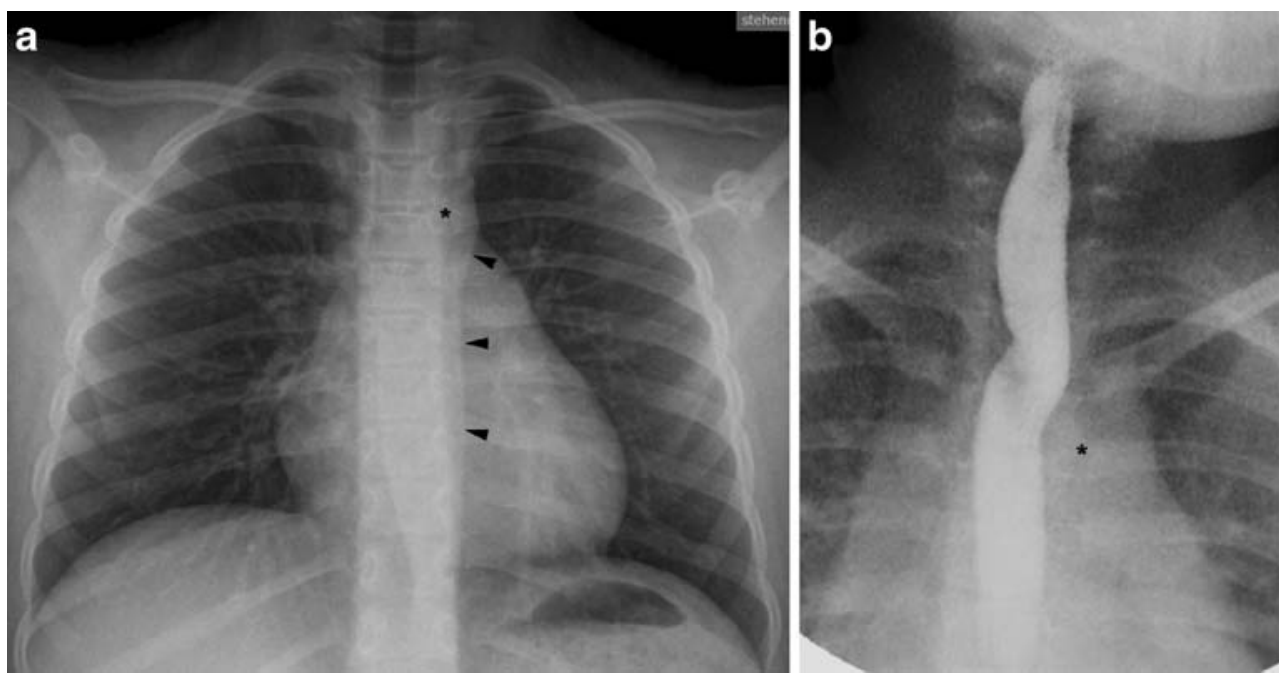

If the left fourth arch regresses distal to the right subclavian artery (A2 or B2), the brachiocephalic vessels originate as a mirror image of a normal left aortic arch. The first arterial branch passes to the left as the left innominate artery, giving rise to the respective common carotid and subclavian arteries followed by the right common carotid and right subclavian arteries. Usually, neither a common left-sided ductus arteriosus, that is generally located anterior to the trachea between left pulmonary artery and left subclavian artery, nor a rare right-sided ductus arteriosus encircle the trachea and oesophagus in combination with a right aortic arch and mirror image branching. However, exceptional cases of vascular rings formed by a right arch with mirror-image branching and left-sided ductus ligament extending from the left pulmonary artery to the right descending aorta (regression at B2) have been described (Fig. 4) [5-7].

If the left fourth aortic arch regresses proximal to the left subclavian artery (C2), a right aortic arch with aberrant left subclavian artery as the last branch results. The aberrant left subclavian artery commonly originates from an aortic diverticulum, passes behind the oesophagus and forms a complete vascular ring together with a left-sided ductus ligament (Fig. 5).

A combination of a right aortic arch and a persisting left descending aorta results in a circumflex right aortic arch with a horizontal retro-oesophageal portion of the dorsal aortic arch (Fig. 6). The ductus arteriosus is more

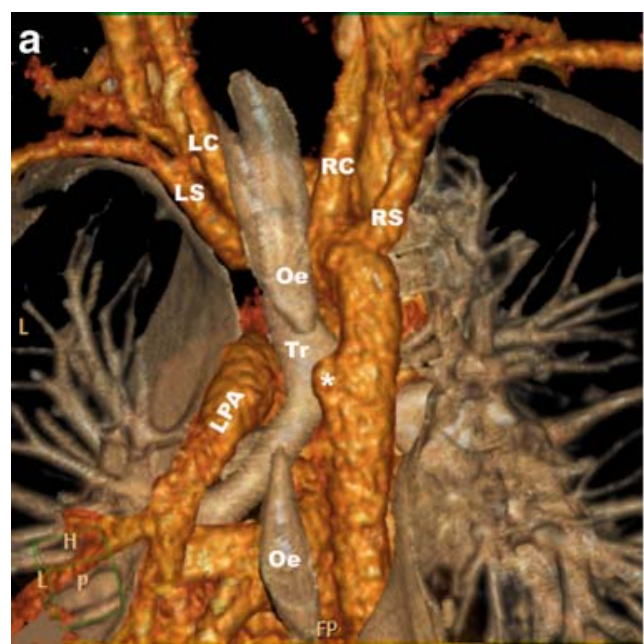

Fig. 4 a, b Vascular ring caused by a right aortic arch with mirrorimage branching and left ductus ligament in a 9-month-old boy with stridor since birth. Volume-rendered contrast-enhanced CT. Posterior view (a) shows the right aortic arch with mirror-image branching of the brachiocephalic arteries to the right of the trachea $(T r)$ and air-filled oesophagus (Oe). The aortic diverticulum (asterisk) of the medial

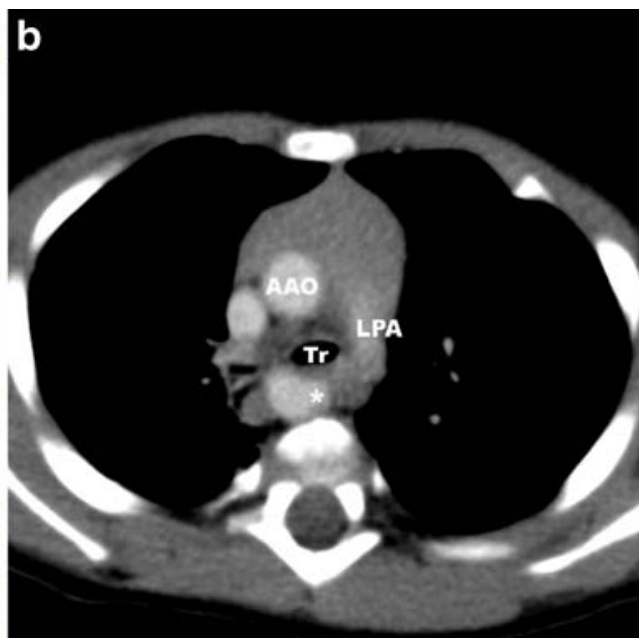

aortic wall implies that there is a left ductus ligament extending to the LPA. Axial contrast-enhanced CT image (b) at the upper border of the LPA confirms a right descending aorta. The ductus ligament is not visualized between the diverticulum (asterisk) and the LPA. Anteroposterior narrowing of the trachea $(T r)$ indicates significant compression by the vascular ring. $A A O$, ascending aorta 


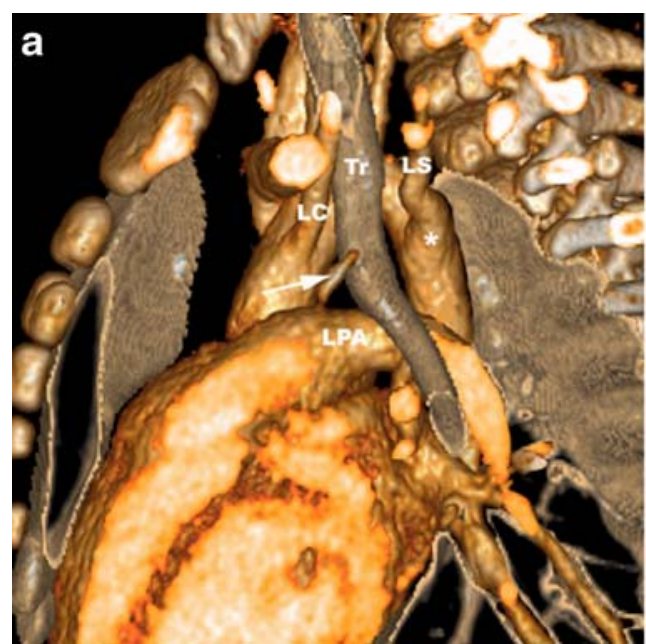

Fig. 5 a, b Right aortic arch with aberrant LS and left ductus ligament in a 2-year-old boy with recurrent respiratory infections. Volume-rendered contrast-enhanced CT. Left lateral view (a) shows mild narrowing and anterior deviation of the trachea $(T r)$ at the level of the calcified left ductus ligament (arrow) that extends from the LPA

commonly located on the left than on the right side, in the former case connecting the left pulmonary artery to the left descending aorta and so completing the vascular ring around the trachea and oesophagus. A right circumflex aorta may have mirror-image branching of the brachiocephalic arteries or an aberrant left subclavian artery, depending on the location of the left fourth arch regression distal (B2) or proximal (C2) to the left subclavian artery.

\section{Double aortic arch}

If both fourth aortic arches persist, two aortic arches encircling the trachea and oesophagus result. Each aortic arch passes over the ipsilateral mainstem bronchus and fuses into the descending aorta, which is more commonly

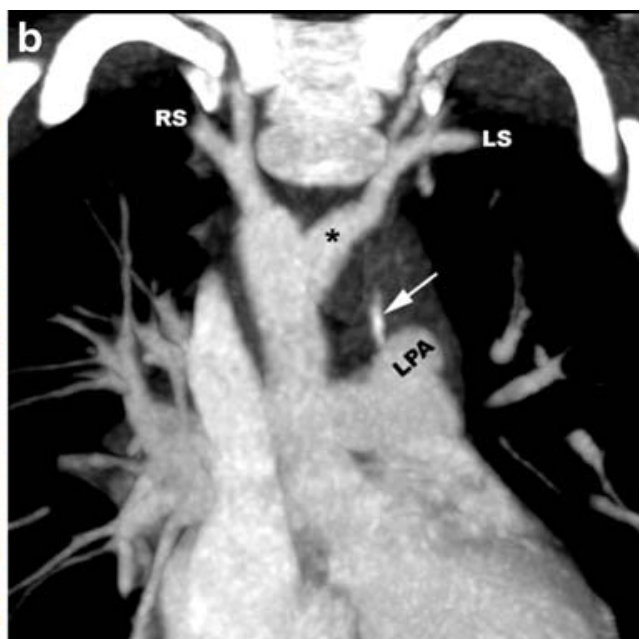

to the aberrant LS originating from an aortic diverticulum (asterisk) This, together with the right aortic arch, completes a vascular ring. Coronal oblique subvolume maximum intensity projection (b) shows high-density calcification of the ductus ligament (arrow)

located on the left than on the right side of the spine. With a left descending aorta, the right arch passes behind the oesophagus and the left arch in front of the trachea. The size of the aortic arches is often asymmetric, usually with the right aortic arch being larger and positioned higher than the left aortic arch (Fig. 7) [8]. A portion of the left aortic arch may be atretic and persist only as a fibrous band (Fig. 8) [9]. The common carotid and subclavian arteries each originate separately from the respective aortic arches. A ductus arteriosus may be present on either or both sides between the respective pulmonary arteries and distal arches.

A double aortic arch, with two patent arches or with an atretic segment of the left aortic arch, is usually an isolated anomaly without associated cardiac malformation and constitutes a tight vascular ring generally producing
Fig. 6 a, b Right circumflex aortic arch in an 8-year-old girl with recurrent respiratory infections. Volume-rendered contrastenhanced MR angiogram. Posterior view (a) shows a right-sided aortic arch with mirror-image branching, retrooesophageal segment, ductus diverticulum (asterisk) implying the presence of a left ductus ligament and a left descending aorta. Axial non-gated steadystate free precession MR images (b) show narrowing of the trachea (arrow) at the level of the retro-oesophageal aortic segment and diverticulum (asterisk)
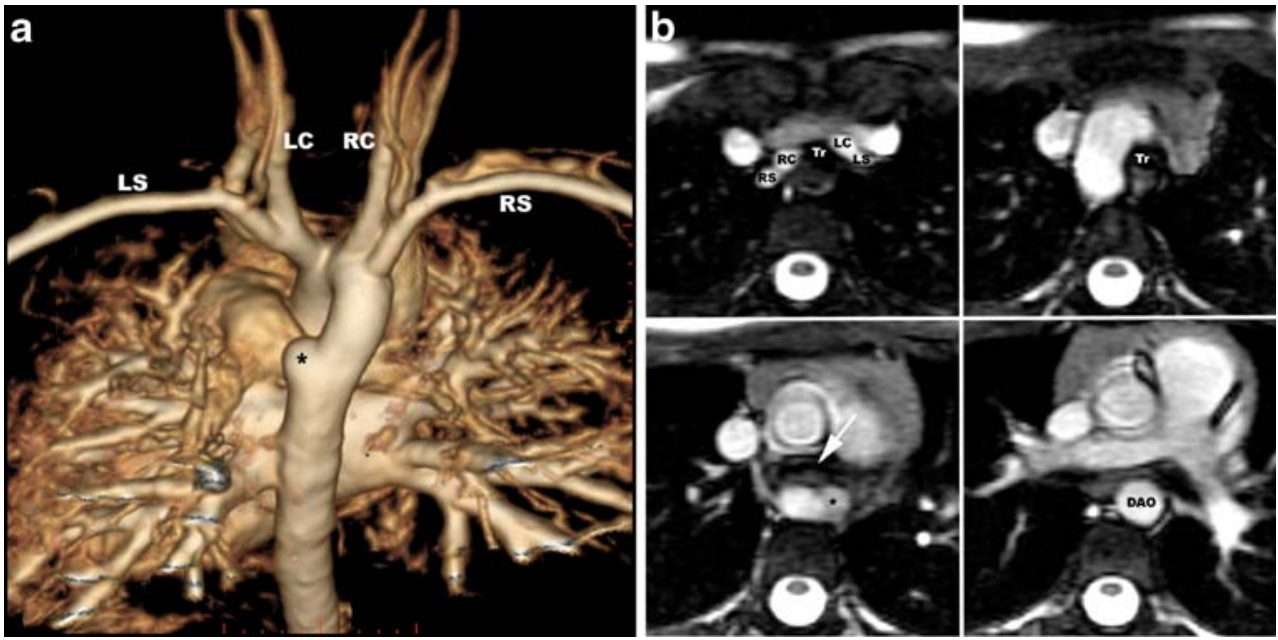


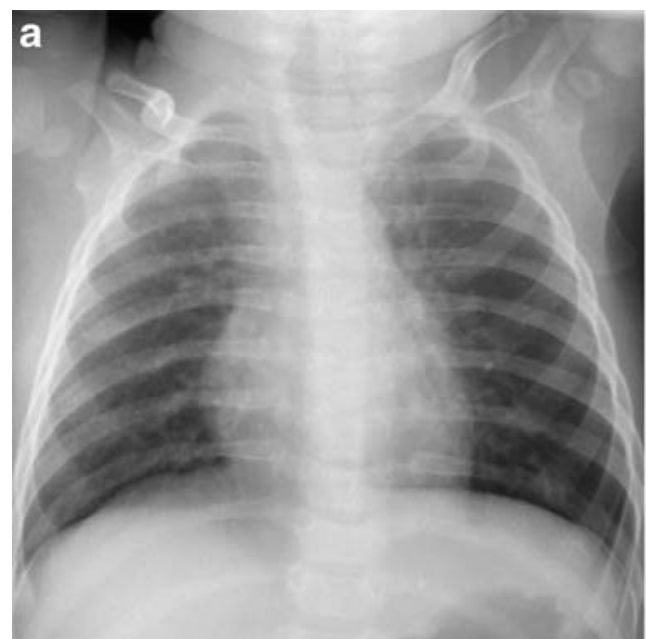

Fig. 7 a, b Double aortic arch in a 5-month-old boy with stridor since birth and recurrent respiratory infections. Frontal chest radiograph (a) shows ill-defined and narrowed midline trachea, the laterality of the aortic arch and descending aorta are not evident. Volume-rendered

symptoms early in infancy (typically stridor, dyspnoea during feeding or recurrent respiratory infections). Surgical division of the smaller or atretic aortic arch and of the ductus ligament is usually required.

\section{Isolated brachiocephalic arteries}

Regression in a single fourth aortic arch at two sites results in isolation of one or more brachiocephalic arteries on the opposite side of the aortic arch, so that they are no longer in continuity with the aorta but attached to the ipsilateral pulmonary artery by the ductus arteriosus $[10,11]$. The isolated arteries are supplied by the vertebral artery (subclavian steal) or collateral arteries from the neck and thorax (Fig. 9). If the ductus arteriosus is patent and pulmonary arterial pressure is normal, there is a left-to-right shunt into the pulmonary artery. Clinically, there may be vertebrobasilar insufficiency or inadequate vascular supply to the ipsilateral arm with decreased pulse pressure.

Isolation of the left subclavian artery (regression at A2 and $\mathrm{C} 2$ ) is more common than that of the right subclavian artery (regression at $\mathrm{A} 1$ and $\mathrm{C} 1$ ) and is often associated with tetralogy of Fallot. Rare cases of isolated left innominate artery (regression at A2 and D2) and left common carotid artery (regression at $\mathrm{C} 2$ and $\mathrm{D} 2$ ) have been described [10].

\section{Interrupted aortic arch}

Interruption of the aortic arch (IAA) results when there is regression of both the right and left fourth aortic arches. The ascending and descending aorta are no longer in continuity and the descending aorta is supplied by a patent ductus arteriosus or collateral arteries when the ductus is closed. The

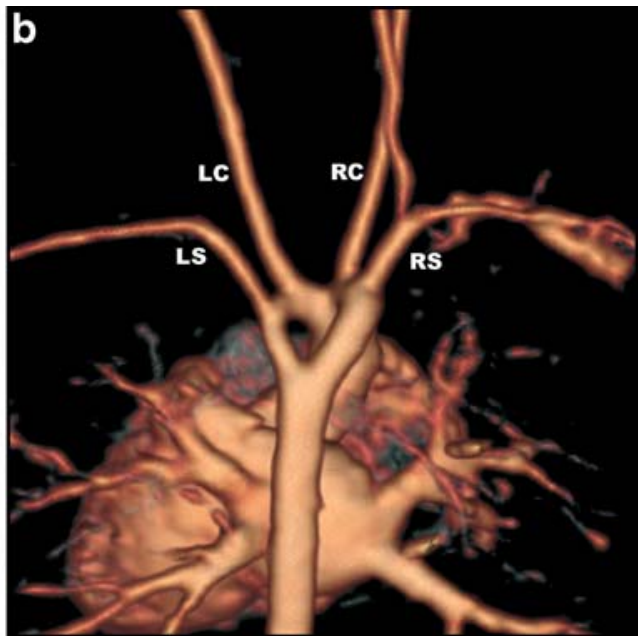

contrast-enhanced MR angiogram. Posterior view (b) shows two patent aortic arches, each giving rise to the respective common carotid $(R C, L C)$ and subclavian $(R S, L S)$ arteries in sequence. The right aortic arch is typically larger and located more superiorly than the left

site of regression can vary in each aortic arch resulting in different anatomic forms. Celoria and Patton [12] have classified IAA into three types depending on the site of interruption: at the aortic isthmus beyond the left subclavian artery (regression at B1and B2) in type A, between the left common carotid and left subclavian arteries (regression at B1 and C2) in type B (Fig. 10), and between both common carotid arteries (regression at B1 and D2) in type C. In each type, the right subclavian artery may arise normally from the innominate artery (regression at B1), may have an aberrant origin as last aortic branch (regression at $\mathrm{C} 1$ ), or may be isolated (regression at B1 and $\mathrm{C} 1$ ) and connected to the pulmonary artery by a patent or ligamentous ductus arteriosus. This classification applies to a "left IAA," but very rarely there may be a mirror-image arrangement of the brachiocephalic arteries and interruption sites corresponding to a "right IAA" $[13,14]$. Type B is the most common form of IAA followed by type $\mathrm{C}$, whereas type $\mathrm{A}$ is rare. Most patients with IAA have a ventricular septum defect and many have other associated cardiac malformations, particularly persistent truncus arteriosus, transposition of the great arteries and aortopulmonary window. A majority of patients with IAA have DiGeorge syndrome (chromosome 22q11.2 deletion syndrome). Newborns with IAA usually present soon after birth with congestive heart failure and respiratory distress when the ductus arteriosus is closing. Currently, surgical correction is attempted during the first week of life [15].

\section{Other abnormalities of the aortic arch system}

A cervical aortic arch is a rare malformation with the apex of the aortic arch extending above the sternum into the neck 
Fig. 8 a-e Double aortic arch with atretic left arch in an 11-year-old boy with stridor on exertion. Frontal chest radiograph (a) shows bilateral paratracheal densities (asterisks) with impression of the trachea from the right side and bilateral paraaortic stripes (arrowheads), the left running over the spine. Lateral chest radiograph (b) shows anterior bowing of the trachea. Barium oesophagography with frontal (c) and lateral (d) projections show broad impressions on the oesophagus from both sides and posteriorly. Volume-rendered contrastenhanced CT, left posterior view (e) shows the close relationship of the LS with the large retrooesophageal diverticulum (asterisk). This appearance suggests atresia of the distal left arch with a fibrotic band tethering the LS and completing the vascular ring
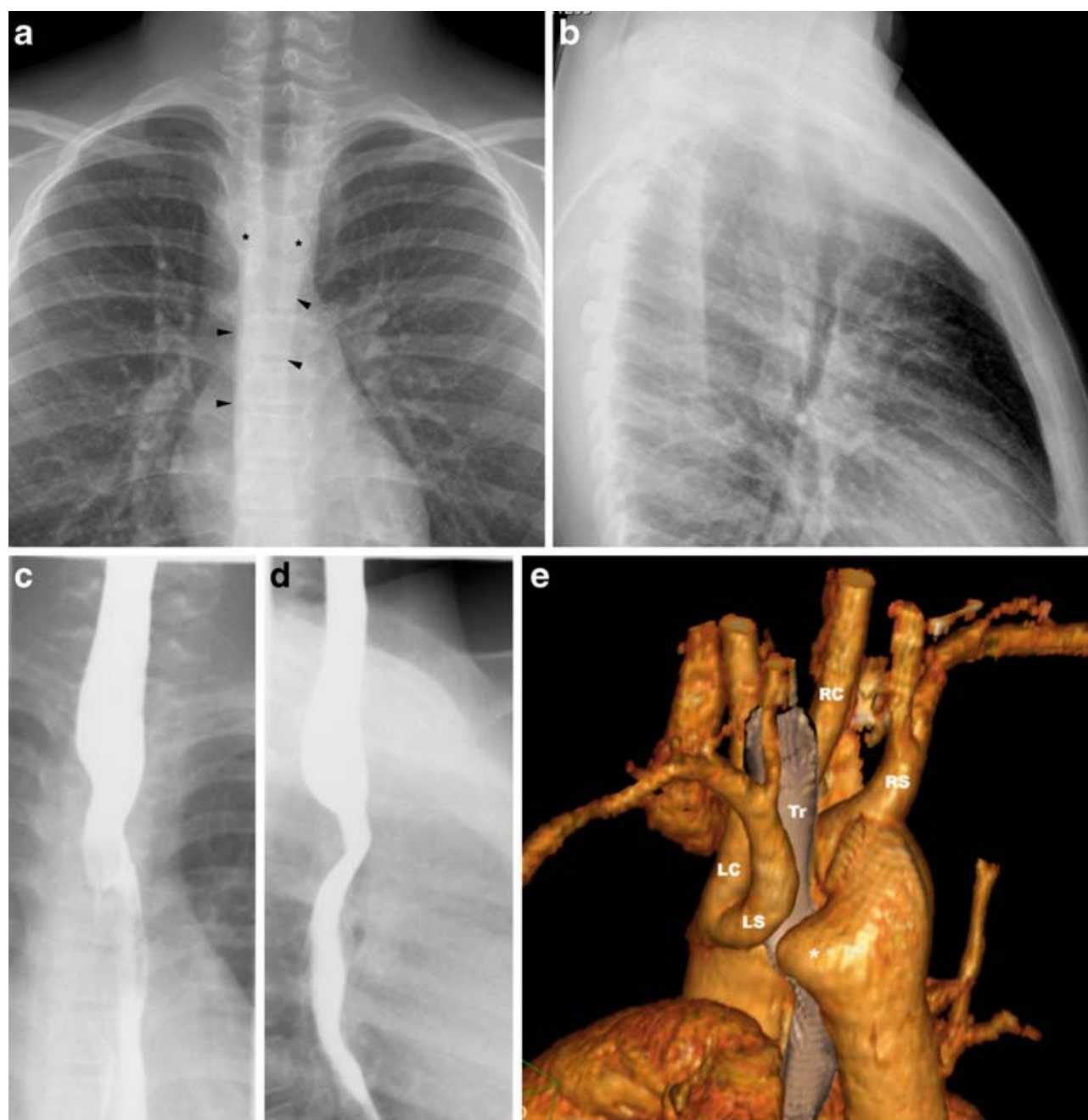

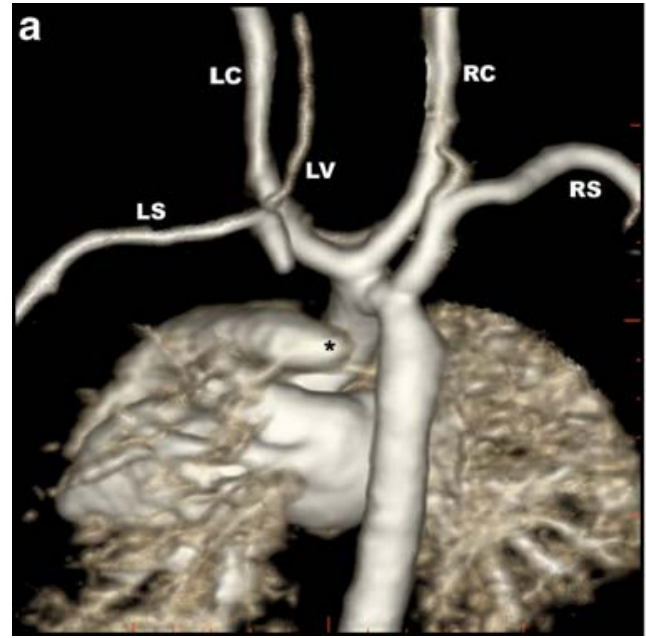

Fig. 9 a, b Isolated LS in a 1-day-old boy. Volume-rendered contrastenhanced MR angiogram. Posterior (a) and right (b) views show a mildly hypoplastic right aortic arch giving rise to the common carotid arteries $(L C, R C)$ with a common origin and the RS in sequence. The LS is isolated, neither connected to an innominate artery or the aortic arch, and is supplied by the left vertebral artery $(L V)$. The left ductus

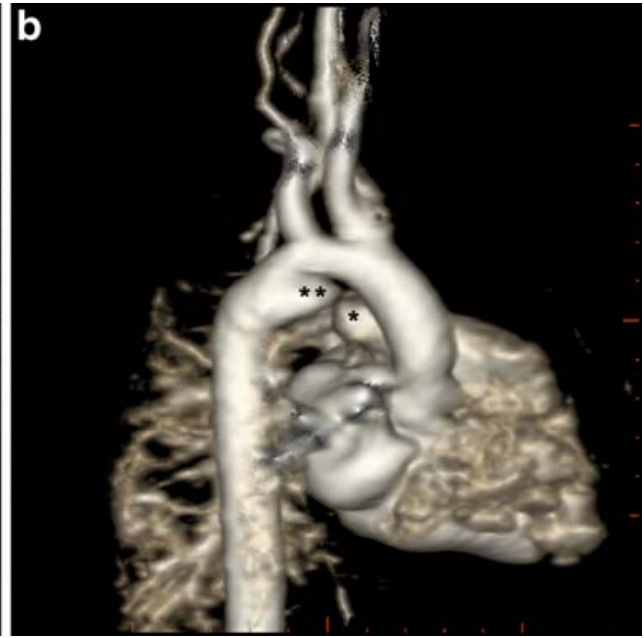

arteriosus, previously connecting the LS to the left pulmonary artery, is completely closed and not visualized. The right ductus arteriosus, extending from the pulmonary artery bifurcation to the aortic isthmus, is partially closed with its proximal (asterisk) and distal (asterisks) portions still visible 

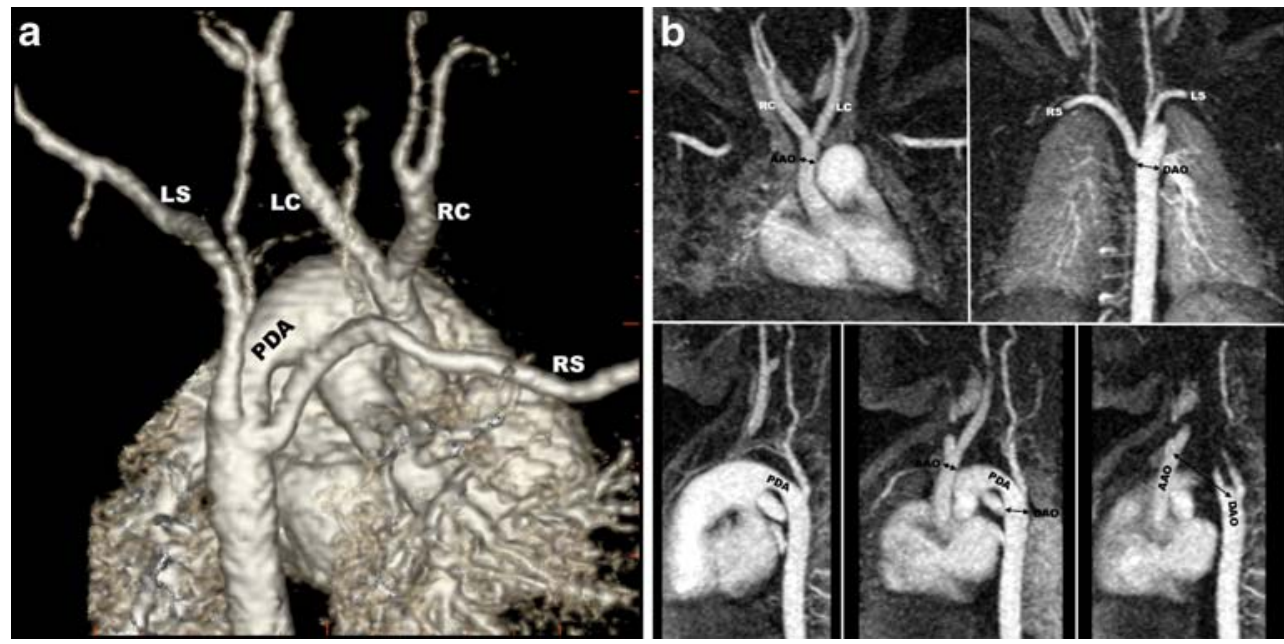

Fig. 10 a, b Interrupted aortic arch type B in a 1-day-old girl. Volume-rendered contrast-enhanced MR angiogram. Right posterior view (a) shows interruption of the aortic arch between the LC and LS arteries. There is an aberrant RS originating as the last branch. The descending aorta $(D A O)$ is supplied by a left patent ductus arteriosus

$[16,17]$. This anomaly is thought to result from persistence of a third aortic arch (more common on the right than on the left side) and regression of the fourth aortic arches. The ipsilateral internal and external carotid arteries may originate directly from the aortic arch. The descending aorta often persists on the contralateral side with a retrooesophageal arch segment and an aberrant subclavian artery arising from an aortic diverticulum. In such a case, a cervical arch forms a complete vascular ring together with the contralateral ductus ligament.

Another very rare malformation is the double-lumen aortic arch, which is formed by a persisting fifth aortic arch and ipsilateral fourth aortic arch giving rise to the brachiocephalic arteries [10, 18]. The persisting fifth aortic arch lies below the true aortic arch connecting the ascending aorta opposite the innominate artery to the distal aortic arch opposite the subclavian artery.

The subclavian artery as first branch of the aorta is a well-recognized entity and has also been explained by persistence of a fifth aortic arch [19, 20]. In combination with regression of the fourth aortic arch and primitive dorsal aorta between the third and fifth aortic arches, the subclavian artery (to the side opposite the definitive aortic arch) originates separately from the distal ascending aorta proximal to the origin of the ipsilateral common carotid artery.

\section{Imaging evaluation}

Clinically silent aortic arch anomalies are usually incidental findings on imaging studies. As some aortic arch anomalies
$(P D A)$. Subvolume maximum intensity projections (b) in coronal oblique (upper row) and sagittal oblique (lower row) planes allow measurement of the dimensions and distance between the ascending aorta $(A A O)$ and descending aorta $(D A O)$

are associated with congenital cardiac malformations, they are often discovered during the imaging evaluation of congenital heart disease. Respiratory distress in a neonate, wheezing, stridor, recurrent respiratory infections and dysphagia may prompt a search for a vascular ring as cause [21]. Chest radiographs and barium oesophagography may affirm the presence of a vascular ring, but crosssectional imaging is usually required to define the exact morphology of the aortic arch malformation and for planning an optimal surgical approach. US allows prenatal diagnosis of aortic arch anomalies and is often sufficient for delineation of the aortic arch, brachiocephalic arteries and ductus arteriosi in neonates due to the large thymus acting as acoustic window [22, 23]. However, complex arch anomalies are better shown and can be fully evaluated with contrast-enhanced helical CT or MR imaging, especially in older children. The multiplanar and three-dimensional (3D) imaging capabilities of CT and MR angiography allow excellent delineation of the aortic arch with its arterial branches and their spatial arrangement. If assessment of the airways is important, CT is currently more reliable than MR for the definition of the airways by multiplanar reconstructions and 3-D rendering with lung window settings [24].

Small or uncooperative children need to be immobilized for the duration of image acquisition both for $\mathrm{CT}$ and MR imaging. For CT imaging, mild sedation is usually sufficient, as the fast scan times achievable with contemporary multidetector scanners allow imaging during quiet breathing without appreciable respiratory artefacts. Alternatively, neonates may be imaged with a "feed-and-wrap" approach without sedation. For MR imaging, children $<8$ years of age require general anaesthesia with controlled 
ventilation, mainly because breath holding is essential for achieving high quality contrast-enhanced angiograms [25]. Although CT may be preferred over MR due to simplicity of the examination and speed of image acquisition, we primarily use MR with contrast-enhanced angiography for the definitive evaluation of an aortic arch anomaly, because it does not involve radiation and we have anaesthesiology support readily available at our institution. Even small vessels of neonates and infants can be imaged excellently with MR, as long as the techniques are adjusted to the small size of the babies [26]. When there is an associated cardiac defect, MR has the advantage to be able to provide additional information on ventricular function and blood flow with electrocardiographically gated cine imaging and flow sensitive sequences. At our institution, we would perform CT for evaluating an aortic arch anomaly only if there is a contraindication to MR imaging, in an unstable patient, or if a congenital thoracic malformation involving the lung parenchyma is suspected. Today, radiographic catheter angiography should no longer be necessary to assess aortic arch malformations.

\section{Radiography}

In symptomatic patients, imaging evaluation usually begins with frontal and lateral chest radiographs $[8,27]$. On the frontal projection, the laterality of the aortic arch can be appreciated by its density and subtle indentation of the trachea (Fig. 3). The trachea may be slightly displaced to the contralateral side of the aortic arch, especially in expiration. With a vascular ring, the trachea will not buckle to the contralateral side, as it is fixed either by the contralateral aortic arch, fibrous arch remnant or ductus ligament. The laterality of the descending aorta can be appreciated by the presence or absence of the para-aortic stripe on the respective side. On the lateral projection, a retro-oesophageal vascular structure can cause an increased retrotracheal density, focal tracheal narrowing or anterior bowing of the distal trachea instead of the normal posterior convexity (Fig. 8) [28].

The presence of a vascular ring should be suspected and further investigated in a symptomatic child when there is a right aortic arch or the arch cannot be located (Fig. 7); or if the trachea is midline, narrowed or bowed anteriorly $[8,28]$.

\section{Barium oesophagography}

Barium oesophagography remains an excellent technique for demonstrating the presence of a vascular ring, but the specific type of ring usually cannot be determined [21, 29]. Vascular structures encircling the oesophagus cause persistent impressions on the barium-filled oesophagus. A single aortic arch may be seen as a lateral impression in older children and adults, but is generally not apparent in infants. A double aortic arch presents with bilateral impressions on the frontal projection and posterior impression on the lateral view (Fig. 8). As the right aortic arch is usually dominant, the right impression is often higher and larger than the left. Broad posterior impressions can be due to a retrooesophageal aortic arch segment (double aortic arch, circumflex aorta) or a large aortic diverticulum. Posterior indentations from aberrant subclavian arteries are smaller and usually also apparent on the frontal projection as an oblique radiolucent band coursing from the side of the aortic arch inferiorly to the opposite side superiorly (Fig. 3).

\section{Cross-sectional imaging with $\mathrm{CT}$ and MR}

The exact anatomy of an aortic arch malformation and its relationship to adjacent structures can be accurately defined by CT and MR [8, 27, 30, 31]. Both methods allow excellent delineation of all patent vessels by contrastenhanced angiography with comparable image quality and the possibility of 3-D display of the malformation. Alternatively, the vessels can be assessed by electrocardiographically gated MR techniques not requiring an intravenous contrast injection, such as spin-echo, cine gradient-echo or steady-state free precession sequences performed in multiple planes. With these MR techniques, narrowing of the trachea can also be appreciated (Fig. 6), but currently multidetector helical CT allows a more detailed 3-D definition of the airways.

Classification of an aortic arch malformation requires a systematic evaluation of following features: 1) laterality of the aortic arch in relation to the trachea, 2) presence, patency and size of bilateral aortic arches, 3) laterality of the proximal descending aorta in relation to the spine, 4) presence and location of an aortic diverticulum and 5) sequence of origins and course of the brachiocephalic arteries.

A complete vascular ring is present when the oesophagus and trachea are encircled by the ascending aorta, aortic arch on one side, and a second aortic arch (patent or fibrotic remnant) or ductus ligament on the contralateral side. A double aortic arch constitutes a tight ring that usually causes symptoms, whereas a ring completed by a contralateral ductus ligament extending to the pulmonary artery is looser and may not be symptomatic. Displacement of the trachea and narrowing of its lumen suggest that there is significant compression by a vascular ring. Although a ductus ligament usually cannot be directly imaged (Fig. 4), its location may be implied by the presence of a diverticulum of the aorta, subclavian artery or pulmonary artery [9]. Such diverticula are caused by the large blood flow volume through the fetal ductus arteriosus. Occasionally, the ductus ligament can be localised on CT when it is calcified (Fig. 5) [32]. The atretic portion of an incomplete 
double aortic arch is also generally not visualized, but the overall 3-D configuration will be suggestive of a double aortic arch (Fig. 8). If there are bilateral aortic arches, the dominant arch needs to be identified. Usually, the arch that is hypoplastic, partially atretic or stenotic will be divided via posterior thoracotomy on the respective side.

Preoperative evaluation of an interrupted aortic arch should include: 1) anatomic type based on the site of interruption and position of the brachiocephalic vessels, 2) patency and laterality of the ductus arteriosus, 3) size of the ascending and descending aorta, 4) distance between the ascending and descending aorta and 5) associated cardiovascular anomalies [33]. The distance between the ascending and descending aorta, as well as their size, determines whether direct anastomosis is possible or if some kind of reconstruction is necessary. The surgeons at our institution insist on 3-D reconstructions of the aorta and its branches, so they can best plan the intervention.

\section{Conclusion}

Familiarity with the spectrum and appearance of possible aortic arch malformations is essential for their accurate diagnosis and classification. Noninvasive imaging with $\mathrm{CT}$ or MR angiography allows complete evaluation of the aortic arch and its branches and provides valuable information for planning surgical management.

\section{References}

1. Amplatz K, Moller JH (1993) Radiology of congenital heart disease. Mosby-Year Book, St. Louis, pp 995-1049

2. Moore KL, Persaud TV (1998) The developing human: clinically oriented embryology. WB Saunders, Philadelphia, pp 384-391

3. Edwards JE (1948) Anomalies of the derivatives of the aortic arch system. Med Clin North Am 32:925-949

4. Edwards JE (1953) Malformations of the aortic arch system manifested as vascular rings. Lab Invest 2:56-75

5. Garti IJ, Aygen MM, Vidne B et al (1973) Right aortic arch with mirror-image branching causing vascular ring. A new classification of the right aortic arch patterns. Br J Radiol 46:115-119

6. Schlesinger AE, Mendeloff E, Sharkey AM et al (1995) MR of right aortic arch with mirror-image branching and a left ligamentum arteriosum: an unusual cause of a vascular ring. Pediatr Radiol 25:455-457

7. Zachary CH, Myers JL, Eggli KD (2001) Vascular ring due to right aortic arch with mirror-image branching and left ligamentum arteriosus: complete preoperative diagnosis by magnetic resonance imaging. Pediatr Cardiol 22:71-73

8. Lowe GM, Donaldson JS, Backer CL (1991) Vascular rings: 10year review of imaging. Radiographics 11:637-646

9. Schlesinger AE, Krishnamurthy R, Sena LM et al (2005) Incomplete double aortic arch with atresia of the distal left arch: distinctive imaging appearance. AJR 184:1634-1639

10. Moes CA, Freedom RM (1993) Rare types of aortic arch anomalies. Pediatr Cardiol 14:93-101
11. Sun AM, Alhabshan F, Branson $\mathrm{H}$ et al (2005) MRI diagnosis of isolated origin of the left subclavian artery from the left pulmonary artery. Pediatr Radiol 35:1259-1262

12. Celoria GC, Patton RB (1959) Congenital absence of the aortic arch. Am Heart J 58:407-413

13. McElhinney DB, Tworetzky W, Hanley FL et al (1999) Congenital obstructive lesions of the right aortic arch. Ann Thorac Surg 67:1194-1202

14. Chu MW, Jutras LF, Tchervenkov CI (2007) Neonatal repair of right interrupted aortic arch, aberrant left subclavian artery, ventricular septal defect and retroaortic innominate vein. Eur J Cardiothorac Surg 31:555-557

15. Mishra PK (2009) Management strategies for interrupted aortic arch with associated anomalies. Eur J Cardiothorac Surg 35:569-576

16. Baravelli M, Borghi A, Rogiani S et al (2007) Clinical, anatomopathological and genetic pattern of 10 patients with cervical aortic arch. Int J Cardiol 114:236-240

17. Haliloglu M, Karcaaltincaba M, Oguz B et al (2007) MR angiography of left-sided cervical aortic arch with aberrant right subclavian artery. Br J Radiol 80:e260-264

18. Yang SG, Fogel MA, Stephens P Jr et al (2003) Noninvasive imaging of isolated persistent fifth aortic arch. Pediatr Cardiol 24:179-181

19. Boechat MI, Gilsanz V, Fellows KE (1978) Subclavian artery as the first branch of the aortic arch: a normal variant in two patients. AJR 131:721-722

20. Moes CA, Benson LN, Burrows PE et al (1991) The subclavian artery as the first branch of the aortic arch. Pediatr Cardiol 12:39-43

21. Bakker DA, Berger RM, Witsenburg M et al (1999) Vascular rings: a rare cause of common respiratory symptoms. Acta Paediatr 88:947-952

22. Yoo SJ, Min JY, Lee YH et al (2003) Fetal sonographic diagnosis of aortic arch anomalies. Ultrasound Obstet Gynecol 22:535-546

23. Lillehei CW, Colan S (1992) Echocardiography in the preoperative evaluation of vascular rings. J Pediatr Surg 27:1118-1120

24. Watanabe N, Hayabuchi Y, Inoue M et al (2009) Tracheal compression due to an elongated aortic arch in patients with congenital heart disease: evaluation using multidetector-row CT. Pediatr Radiol 39:1048-1053

25. Saleh RS, Patel S, Lee MH et al (2007) Contrast-enhanced MR angiography of the chest and abdomen with use of controlled apnea in children. Radiology 243:837-846

26. Kellenberger CJ, Yoo SJ, Büchel ER (2007) Cardiovascular MR imaging in neonates and infants with congenital heart disease. Radiographics 27:5-18

27. Oddone M, Granata C, Vercellino N et al (2005) Multi-modality evaluation of the abnormalities of the aortic arches in children: techniques and imaging spectrum with emphasis on MRI. Pediatr Radiol 35:947-960

28. Pickhardt PJ, Siegel MJ, Gutierrez FR (1997) Vascular rings in symptomatic children: frequency of chest radiographic findings. Radiology 203:423-426

29. Turner A, Gavel G, Coutts J (2005) Vascular rings-presentation, investigation and outcome. Eur J Pediatr 164:266-270

30. Lee EY, Siegel MJ, Hildebolt CF et al (2004) MDCT evaluation of thoracic aortic anomalies in pediatric patients and young adults: comparison of axial, multiplanar, and 3D images. AJR 182:777-784

31. Eichhorn J, Fink C, Delorme S et al (2004) Rings, slings and other vascular abnormalities. Ultrafast computed tomography and magnetic resonance angiography in pediatric cardiology. Z Kardiol 93:201-208

32. Bisceglia M, Donaldson JS (1991) Calcification of the ligamentum arteriosum in children: a normal finding on CT. AJR $156: 351-352$

33. Yang DH, Goo HW, Seo DM et al (2008) Multislice CT angiography of interrupted aortic arch. Pediatr Radiol 38:89-100 